\title{
The Origins Space Telescope
}

\section{Leisawitz, E. Amatucci, L. Allen, J. Arenberg, L. Armus, et al.}

D. Leisawitz, E. Amatucci, L. Allen, J. Arenberg, L. Armus, C. Battersby, B. G. Beaman, J. Bauer, R. Bell, P. Beltran, D. Benford, E. Bergin, J. Bolognese, C. M. Bradford, D. Bradley, D. Burgarella, S. Carey, R. Carter, J. D. Chi, A. Cooray, J. Corsetti, T. D'Asto, E. De Beck, K. Denis, C. Derkacz, L. Dewell, M. DiPirro, C. P. Earle, M. East, S. Edgington, K. Ennico, L. Fantano, G. Feller, A. Flores, D. Folta, J. Fortney, B. J. Gavares, J. Generie, M. Gerin, Z. Granger, T. P. Greene, A. Griffiths, G. Harpole, K. Harvey, F. Helmich, G. Helou, L. Hilliard, J. Howard, M. Jacoby, A. Jamil, T. Jamison, L. Kaltenegger, T. Kataria, J. S. Knight, P. Knollenberg, C. Lawrence, P. Lightsey, S. Lipscy, C. Lynch, E. Mamajek, G. Martins, J. C. Mather, M. Meixner, G. Melnick, S. Milam, T. Mooney, S. H. Moseley, D. Narayanan, S. Neff, T. Nguyen, A. Nordt, J. Olson, D. Padgett, M. Petach, S. Petro, J. Pohner, K. Pontoppidan, A. Pope, D. Ramspacher, A. Rao, G. Rieke, M. Rieke, T. Roellig, I. Sakon, C. Sandin, K. Sandstrom, D. Scott, L. Seals, K. Sheth, J. Staguhn, J. Steeves, K. Stevenson, L. Stokowski, E. Stoneking, K. Su, K. Tajdaran, S. Tompkins, J. Turner, J. Vieira, C. Webster, M. Wiedner, E. L. Wright, C. Wu, J. Zmuidzinas, "The Origins Space Telescope," Proc. SPIE 11115, UV/ Optical/IR Space Telescopes and Instruments: Innovative Technologies and Concepts IX, 111150Q (9 September 2019); doi: 10.1117/12.2530514

Event: SPIE Optical Engineering + Applications, 2019, San Diego, California, United States 


\section{The Origins Space Telescope}

D. Leisawitz ${ }^{\mathrm{a}}$ E. Amatucci ${ }^{\mathrm{a}}$, L. Allen ${ }^{\mathrm{b}}$, J. Arenberg ${ }^{\mathrm{c}}$, L. Armus ${ }^{\mathrm{d}}$, C. Battersby ${ }^{\mathrm{e}}$, B.G. Beaman ${ }^{\mathrm{a}, \text { f }}$, J. Bauer $^{\mathrm{g}}$, R. Bell ${ }^{\mathrm{h}}$, P. Beltran ${ }^{\mathrm{a}}$, D. Benford ${ }^{\mathrm{i}}$, E. Bergin', J. Bolognese ${ }^{\mathrm{a}}$, C.M. Bradford ${ }^{\mathrm{j}}$, D. Bradley ${ }^{\mathrm{a}}$, D.

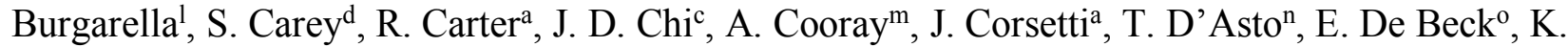

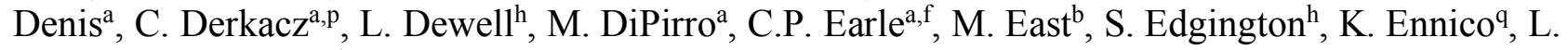
Fantano $^{\mathrm{a}}$, G. Feller ${ }^{\mathrm{h}}$, A. Flores ${ }^{\mathrm{a}}$, D. Folta ${ }^{\mathrm{a}}$, J. Fortney ${ }^{\mathrm{r}}$, B.J. Gavares ${ }^{\mathrm{a}, \mathrm{s}}$, J. Generie ${ }^{\mathrm{a}}$, M. Gerin ${ }^{\mathrm{t}}$, Z. Granger $^{\mathrm{h}}$, T.P. Greene ${ }^{\mathrm{q}}$, A. Griffiths ${ }^{\mathrm{u}}$, G. Harpole ${ }^{\mathrm{c}}$, K. Harvey ${ }^{\mathrm{b}}$, F. Helmich ${ }^{\mathrm{v}}$, G. Helou ${ }^{\mathrm{d}}$, L. Hilliard ${ }^{\mathrm{a}}$, J. Howard ${ }^{\mathrm{a}}$, M. Jacoby ${ }^{\mathrm{h}}$, A. Jamil ${ }^{\mathrm{a}}$, T. Jamison ${ }^{\mathrm{a}}$, L. Kaltenegger ${ }^{\mathrm{w}}$, T. Kataria ${ }^{\mathrm{k}}$, J.S. Knight ${ }^{\mathrm{x}}$, P. Knollenberg ${ }^{\mathrm{c}}$, C. Lawrence ${ }^{\mathrm{k}}$, P. Lightsey ${ }^{\mathrm{x}}$, S. Lipscy ${ }^{\mathrm{x}}$, C. Lynch ${ }^{\mathrm{a}, \mathrm{p}}$, E. Mamajek ${ }^{\mathrm{k}}$, G. Martins ${ }^{\mathrm{a}}$, J.C. Mather $^{\mathrm{a}}$, M. Meixner ${ }^{\mathrm{y}, \mathrm{z}}$, G. Melnick ${ }^{\mathrm{A}}$, S. Milam ${ }^{\mathrm{a}}$, T. Mooney ${ }^{\mathrm{b}}$, S.H. Moseley ${ }^{\mathrm{a}}$, D. Narayanan ${ }^{\mathrm{B}}, \mathrm{S}$.

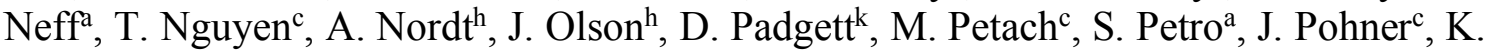

Pontoppidan $^{z}$, A. Pope ${ }^{\mathrm{C}}$, D. Ramspacker ${ }^{\mathrm{a}}$, A. Rao ${ }^{\mathrm{a}}$, G. Rieke ${ }^{\mathrm{D}}$, M. Rieke ${ }^{\mathrm{D}}$, T. Roellig' ${ }^{\mathrm{q}}$ I. Sakon ${ }^{\mathrm{E}}$, C. Sandin $^{\mathrm{a}}$, K. Sandstrom ${ }^{\mathrm{F}}$, D. Scott ${ }^{\mathrm{G}}$, L. Seals ${ }^{\mathrm{a}}$, K. Sheth ${ }^{\mathrm{i}}$, J. Staguhn ${ }^{\mathrm{a}, \mathrm{y}}$, J. Steeves ${ }^{\mathrm{k}}$, K. Stevenson ${ }^{\mathrm{z}}$, L. Stokowski $^{\text {h }}$ E. Stoneking ${ }^{\mathrm{a}}, \mathrm{K} . \mathrm{Su}^{\mathrm{D}}$, K. Tajdaran ${ }^{\mathrm{h}}$, S. Tompkins ${ }^{\mathrm{a}}$, J. Turner ${ }^{\mathrm{H}}$, J. Vieira ${ }^{\mathrm{I}}$, C. Webster ${ }^{\mathrm{a}}$, M. Wiedner ${ }^{\mathrm{t}}$, E.L. Wright ${ }^{\mathrm{H}}, \mathrm{C} . \mathrm{Wu}^{\mathrm{a}}$, and J. Zmuidzinas ${ }^{\mathrm{J}}$

${ }^{a}$ NASA Goddard Space Flight Center, 8800 Greenbelt Rd., Greenbelt, MD 20771 USA

${ }^{\mathrm{b}}$ Harris Corporation, Melbourne, FL, USA

${ }^{c}$ Northrop-Grumman Aerospace Systems, Redondo Beach, CA, USA

${ }^{\mathrm{d}}$ Caltech/Infrared Processing and Analysis Center, Pasadena, CA, USA

e Department of Physics, University of Connecticutt, Storrs, CT, USA

${ }^{\mathrm{f}}$ Science Systems and Applications, Inc., Lanham, MD, USA

g Astronomy Department, University of Maryland, College Park, MD, USA

${ }^{\mathrm{h}}$ Lockheed-Martin Advanced Technology Center, Palo Alto, CA, USA

${ }^{\text {i } A s t r o p h y s i c s ~ D i v i s i o n, ~ S c i e n c e ~ M i s s i o n ~ D i r e c t o r a t e, ~ N A S A ~ H e a d q u a r t e r s, ~ W a s h i n g t o n, ~ D C, ~ U S A ~}$

j Department of Astronomy, University of Michigan, Ann Arbor, MI, USA

${ }^{\mathrm{k}}$ Caltech/Jet Propulsion Laboratory, Pasadena, CA, USA

${ }^{1}$ Laboratoire d'Astrophysique de Marseille, Marseille, France

${ }^{m}$ Department of Physics and Astronomy, University of California, Irvine, Irvine, CA, USA

${ }^{n}$ ATA Aerospace Civil, Greenbelt, MD, USA

${ }^{\circ}$ Dept. of Space, Earth and Environment, Chalmers Institute of Technology, Gothenburg, Sweden

p InuTeq, LLC, Beltsville, MD, USA

q NASA Ames Research Center, Mountain View, CA, USA

${ }^{\mathrm{r}}$ Astronomy and Astrophysics Dept., University of California, Santa Cruz, Santa Cruz, CA, USA

${ }^{s}$ Orbital ATK, Dulles, VA, USA

${ }^{t}$ Sorbonne Université, Observatoire de Paris, CNRS, LERMA, F-75014, Paris, France

u University of Nottingham,

${ }^{v}$ Netherlands Institute for Space Research (SRON), Groningen, The Netherlands

${ }^{w}$ Cornell University, Ithaca, NY

x Ball Aerospace Corporation, Boulder, CO, USA

y Johns Hopkins University, Baltimore, MD, USA

${ }^{z}$ Space Telescope Science Institute, Baltimore, MD, USA

${ }^{A}$ Harvard-Smithsonian Center for Astrophysics, Cambridge, MA, USA

B Department of Astronomy, University of Florida, Gainesville, FL, USA

${ }^{\mathrm{C}}$ Department of Astronomy, University of Massachusetts, Amherst, MA, USA

UV/Optical/IR Space Telescopes and Instruments: Innovative Technologies and Concepts IX, edited by Allison A. Barto, James B. Breckinridge, H. Philip Stahl, Proc. of SPIE Vol. 11115, 111150Q · (c) 2019 SPIE CCC code: $0277-786 X / 19 / \$ 21 \cdot$ doi: $10.1117 / 12.2530514$ 
D Dept. of Astronomy and Steward Observatory, The University of Arizona, Tucson, AZ, USA

E School of Science, The University of Tokyo, 7-3-1 Hongo, Bunkyo-ku, Tokyo, Japan

F CASS, University of California, San Diego, San Diego, CA, USA

G Dept. of Physics and Astronomy, The University of British Columbia, Vancouver, BC, Canada

${ }^{\mathrm{H}}$ Division of Astronomy and Astrophysics, UCLA, Los Angeles, CA, USA

I Department of Astronomy, University of Illinois, Urbana-Champaign, Urbana, IL, USA

${ }^{\mathrm{J}}$ Division of Physics, Mathematics and Astronomy, Caltech, Pasadena, CA, USA

\begin{abstract}
The Origins Space Telescope will trace the history of our origins from the time dust and heavy elements permanently altered the cosmic landscape to present-day life. How did galaxies evolve from the earliest galactic systems to those found in the universe today? How do habitable planets form? How common are life-bearing worlds? To answer these alluring questions, Origins will operate at mid- and far-infrared wavelengths and offer powerful spectroscopic instruments and sensitivity three orders of magnitude better than that of Herschel, the largest telescope flown in space to date.

After a $3 \frac{1}{2}$ year study, the Origins Science and Technology Definition Team will recommend to the Decadal Survey a concept for Origins with a 5.9-m diameter telescope cryocooled to $4.5 \mathrm{~K}$ and equipped with three scientific instruments. A mid-infrared instrument (MISC-T) will measure the spectra of transiting exoplanets in the $2.8-20 \mu \mathrm{m}$ wavelength range and offer unprecedented sensitivity, enabling definitive biosignature detections. The Far-IR Imager Polarimeter (FIP) will be able to survey thousands of square degrees with broadband imaging at 50 and $250 \mu \mathrm{m}$. The Origins Survey Spectrometer (OSS) will cover wavelengths from $25-588 \mu \mathrm{m}$, make wide-area and deep spectroscopic surveys with spectral resolving power $\mathrm{R} \sim 300$, and pointed observations at $\mathrm{R} \sim 40,000$ and 300,000 with selectable instrument modes. Origins was designed to minimize complexity. The telescope has a Spitzer-like architecture and requires very few deployments after launch. The cryo-thermal system design leverages JWST technology and experience. A combination of current-state-of-the-art cryocoolers and next-generation detector technology will enable Origins' natural backgroundlimited sensitivity.
\end{abstract}

\title{
1. INTRODUCTION
}

This paper is based on the Executive Summary of the Origins Space Telescope study team's report to the 2020 Astrophysics Decadal Survey (see https://asd.gsfc.nasa.gov/firs/docs/)

The Origins Space Telescope (Origins; Fig. 1) traces our cosmic history from the formation of the first galaxies and the rise of metals and dust to the development of habitable worlds and present-day life. Origins does this through exquisite sensitivity to infrared radiation from ions, atoms, molecules, dust, water vapor and ice, and observations of extra-solar

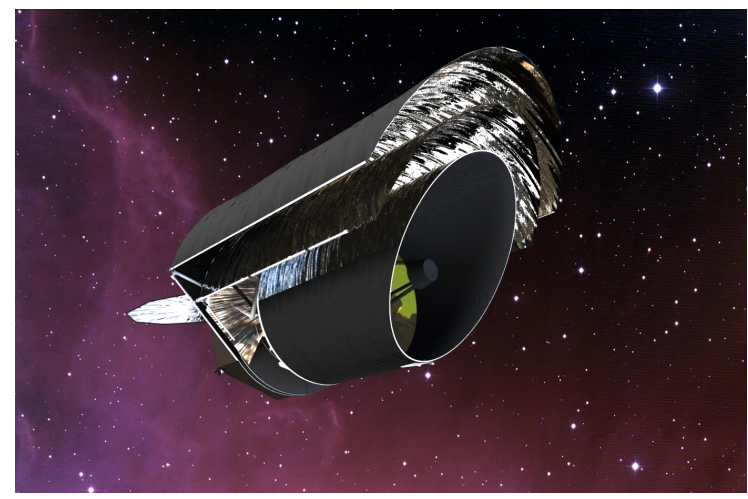

Figure 1. The Origins concept is low-risk and 1000 times more sensitive than prior far-IR missions. With an aperture diameter of $5.9 \mathrm{~m}$ and a suite of powerful instruments, Origins operates with spectral resolving power from 3 to $3 \times 10^{5}$ over the wavelength range $2.8-588 \mu \mathrm{m}$. The Origins design has very few critical deployments and builds upon the technical heritage of Spitzer, with passive cooling from a two-layer sunshield and advanced cryocoolers maintaining the telescope and instruments at $4.5 \mathrm{~K}$. Origins is agile, enabling $>80 \%$ observing efficiency, in line with the $90 \%$ efficiency achieved with Herschel. 
planetary atmospheres, protoplanetary disks, and large-area extragalactic fields. Origins operates in the wavelength range 2.8 to $588 \mu \mathrm{m}$ and owes its superlative sensitivity to a large, cold $(4.5 \mathrm{~K})$ telescope and advanced instruments.

Origins investigates the creation and dispersal of elements essential to life, the formation of planetary systems and the transport of water to habitable worlds, and the atmospheres of exoplanets around nearby K- and M-dwarfs to identify potentially habitable — and even inhabited — worlds. These science priorities are motivated by their profound significance, as well as expected advances from, and limitations of, current and next generation observatories (JWST, WFIRST, ALMA, LSST). The nine key Origins scientific objectives (Table 1) address NASA's three major astrophysics science goals: How does the Universe work? How did we get here? and Are we alone?

\begin{tabular}{|c|c|c|c|}
\hline NASA Goal & How Does the Universe Work? & How Did We Get Here? & Are We Alone? \\
\hline $\begin{array}{l}\text { Origins } \\
\text { Science } \\
\text { Goals }\end{array}$ & $\begin{array}{l}\text { How do galaxies form stars, } \\
\text { make metals, and grow their } \\
\text { central supermassive black holes } \\
\text { from reionization to today? }\end{array}$ & $\begin{array}{l}\text { How do the conditions for } \\
\text { habitability develop during } \\
\text { the process of planet } \\
\text { formation? }\end{array}$ & $\begin{array}{l}\text { Do planets orbiting K \& M-dwarf } \\
\text { stars support life? }\end{array}$ \\
\hline $\begin{array}{l}\text { Origins } \\
\text { Scientific } \\
\text { Capabilities }\end{array}$ & $\begin{array}{l}\text { Using sensitive spectroscopic capabilities of a } \\
\text { cold telescope, Origins will measure properties } \\
\text { of star- formation and growing black holes in } \\
\text { galaxies across all epochs. }\end{array}$ & $\begin{array}{l}\text { With sensitive, high-resolution } \\
\text { spectroscopy, Origins will illuminate } \\
\text { the path of water and its abundance to } \\
\text { determine the availability of water for } \\
\text { habitable planets. }\end{array}$ & $\begin{array}{l}\text { By obtaining precise mid-infrared transmission } \\
\text { and emission spectra, Origins will assess the } \\
\text { habitability of nearby exoplanets and search for } \\
\text { signs of life. }\end{array}$ \\
\hline $\begin{array}{l}\text { Origins } \\
\text { Scientific } \\
\text { Objectives }\end{array}$ & $\begin{array}{l}\text { 1. How does the relative growth of stars and } \\
\text { supermassive black holes in galaxies evolve } \\
\text { with time? } \\
\text { 2. How do galaxies make metals, dust, and } \\
\text { organic molecules? } \\
\text { 3. How do the relative energetics from super- } \\
\text { novae and quasars influence the interstellar } \\
\text { medium of galaxies? }\end{array}$ & $\begin{array}{l}\text { 1. What role does water play in the forma- } \\
\text { tion and evolution of habitable planets? } \\
\text { 2. How and when do planets form? } \\
\text { 3. How were water and life's ingredients } \\
\text { delivered to Earth and to exoplanets? }\end{array}$ & $\begin{array}{l}\text { 1. What fraction of terrestrial planet around M- } \\
\text { and K-dwarf stars has tenuous, clear, or cloudy } \\
\text { atmospheres? } \\
\text { 2. What fraction of terrestrial M-dwarf planets is } \\
\text { temperate? } \\
\text { 3. What types of temperate, terrestrial, M-dwarf } \\
\text { planets support life? }\end{array}$ \\
\hline
\end{tabular}

These scientific objectives drive the instrumental requirements shown in Table 2. The concept study report includes a comprehensive Science Traceability Matrix. The Origins design is powerful and versatile, and the infrared emission it detects is information-rich. Origins will enable astronomers in the 2030s to ask new questions not yet imagined, and provide a far-infrared window (Fig. 2) complementary to planned, next-generation observatories, such as LISA, Athena, and ground-based ELTs.

\begin{tabular}{|c|c|c|c|c|c|}
\hline \multicolumn{6}{|c|}{$\begin{array}{l}\text { Table 2: Summary of Origins Requirements } \\
\text { (Full Scientific Traceability Matrix (STM) provided in Table 1.5-1) }\end{array}$} \\
\hline \multicolumn{2}{|c|}{ Origins Science Driver } & \multicolumn{4}{|c|}{ Technical or Instrument Parameter } \\
\hline Scientific Goal & Observable & Parameter & Requirement & Design & Scientific Impact with Anticipated Changes \\
\hline $\begin{array}{l}\text { How do } \\
\text { galaxies form }\end{array}$ & \multirow{2}{*}{$\begin{array}{l}\text { Mid- and far- } \mathbb{R} \\
\text { rest-frame spectral } \\
\text { lines. }\end{array}$} & $\begin{array}{l}\text { Aperture } \\
\text { Size }\end{array}$ & $3.0-5.0 \mathrm{~m}$ & $5.9 \mathrm{~m}$ & $\begin{array}{l}>3.0 \mathrm{~m} \text { based on the angular resolution needed to study } \\
\text { high- } z \text { galaxies. }>5.0 \mathrm{~m} \text { driven by } z>6 \text { galaxy studies. }\end{array}$ \\
\hline $\begin{array}{l}\text { stars, make } \\
\text { metals, and } \\
\text { ir central SMBHs? }\end{array}$ & & $\begin{array}{c}\text { Aperture } \\
\text { Temperature }\end{array}$ & $<6 K$ & $4.5 \mathrm{~K}$ & $\begin{array}{l}\text { Sufficiently cold temperature to meet the sensitivity } \\
\text { requirements at the longest wavelengths. } ._{\text {tel }}>6 \mathrm{~K} \text { impacts } \\
\text { the ability to conduct sciences }>300 \mu \mathrm{m} \text {. }\end{array}$ \\
\hline \multirow{4}{*}{$\begin{array}{l}\text { How do the } \\
\text { conditions for } \\
\text { habitability } \\
\text { develop during } \\
\text { the process of planet } \\
\text { formation? }\end{array}$} & $\begin{array}{l}\mathrm{H}_{2}^{18} \mathrm{O}_{110-101} \text { at } \\
547.4-1 \mu \mathrm{m}\end{array}$ & $\lambda_{\max }$ & $>550 \mu \mathrm{m}$ & $588 \mu \mathrm{m}$ & $\begin{array}{l}\mathrm{H}_{2} 0 \text { 110-101 is at } 538.3-\mu \mathrm{m} ; \lambda_{\max }<538 \mu \mathrm{m} \text { impacts water } \\
\text { sciences. } \lambda_{\max }<500 \mu \mathrm{m} \text { impacts extragalactic sciences. }\end{array}$ \\
\hline & $\begin{array}{c}\mathrm{H}_{2} \mathrm{O} 212-101179.5- \\
\text { um line }\end{array}$ & $R=N / \Delta \lambda$ & 200,000 & 202,785 & Smaller spectral resolution impacts doppler tomography. \\
\hline & \multirow[t]{2}{*}{$\begin{array}{l}\text { HD 1-0 112- }-\mu m \\
\quad \text { line }\end{array}$} & $\begin{array}{l}\text { Spectral line } \\
\text { sensitivity }\end{array}$ & $\begin{array}{c}10^{-20} \mathrm{~W} \mathrm{~m}^{-2}(1 \\
\mathrm{hr} ; 50)\end{array}$ & $\begin{array}{c}5 \times 10^{-21} \mathrm{~W} \mathrm{~m}^{-2} \\
(1 \mathrm{hr} ; 50)\end{array}$ & $\begin{array}{l}\text { Below this sensitivity, Origins cannot study sufficient disks } \\
\text { at the distance of Orion for gas mass measurements. }\end{array}$ \\
\hline & & $R=N / \Delta \lambda$ & 40,000 & 43,000 & Lowering R reduces the gas mass accuracy. \\
\hline \multirow{2}{*}{$\begin{array}{l}\text { Do planets } \\
\text { orbiting } \\
\text { M-dwarf stars } \\
\text { support life? }\end{array}$} & \multirow{2}{*}{$\begin{array}{c}\mathrm{CH}_{4}(3.3 \& 7.4 \mu \mathrm{m}), \\
\mathrm{N}_{0} \mathrm{O}(4.5 \& 7.8 \mu \mathrm{m}) \\
\mathrm{O}_{3}(9.7 \mu \mathrm{m}), \mathrm{CO} \\
(4.3 \& 15 \mu \mathrm{m}), \mathrm{H}_{2} \mathrm{O} \\
(6.3,17+\mu \mathrm{m})^{2}\end{array}$} & $\lambda \min$ & $<3 \mu \mathrm{m}$ & $2.8 \mu \mathrm{m}$ & $\begin{array}{l}\mathrm{CO}_{2} \text { at } 4.3 \mu \mathrm{m} \text { strongest of all features; } \lambda_{\min }>5 \mu \mathrm{m} \text { reduces } \\
\text { the exoplanet case to surface temperature only. }\end{array}$ \\
\hline & & $\begin{array}{l}\text { Aperture } \\
\text { Size }\end{array}$ & $5.3 \mathrm{~m}$ & $5.9 m$ & $\begin{array}{l}\text { An aperture size }<5.3 \mathrm{~m} \text { results in a dramatic drop in the } \\
\text { ability to detect } \mathrm{CH}_{4} \text { and } \mathrm{N}_{2} \mathrm{O} \text { - crucial biosignatures - in } \\
\text { exoplanet transits over a } 5 \text {-vear mission. }\end{array}$ \\
\hline
\end{tabular}




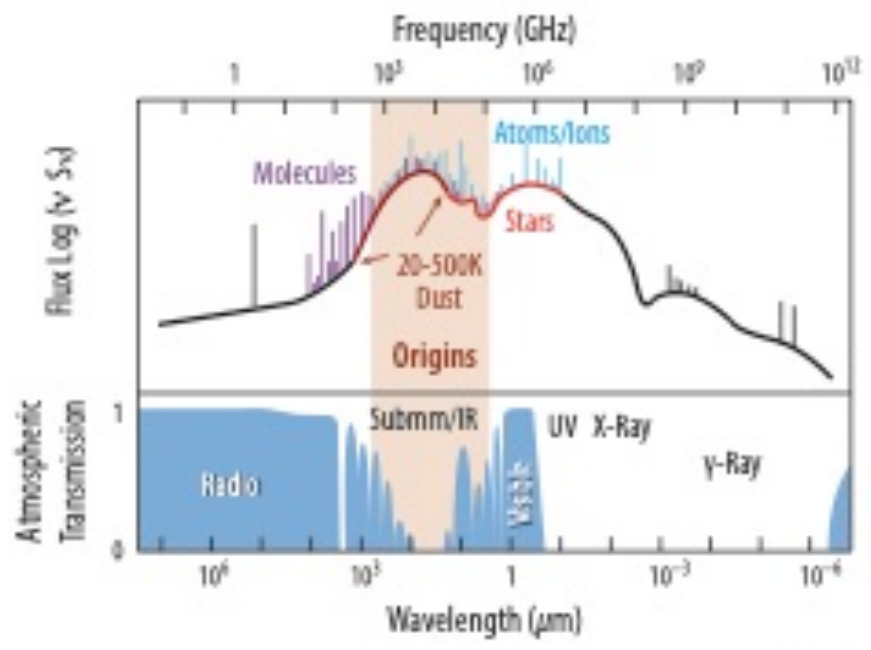

Figure 2. Origins studies the Universe at a wavelength range that is inaccessible from the ground. Top: The spectrum of a typical galaxy. Bottom Infrared photons between 2.8 and $588 \mu \mathrm{m}$ in Origins, wavelength range capture emission from stars, molecules, dust, and ions/atoms, enabling a multipronged probe into key physical processes in galaxies.

\section{SCIENCE GOALS AND OBJECTIVES}

\subsection{How do galaxies form stars, make metals, and grow their central supermassive black holes, from the Epoch of Reionization to today?}

Decades of observations have shown that galaxies condensed out of primordial gas, built up their stellar mass, heavy metals, and central supermassive black holes (SMBHs), and evolved into the systems we see today. Yet we still do not understand how this happened. There is a rich interplay between the drivers of galaxy evolution, which can only be understood through new observations in a currently inaccessible wavelength regime, the far-infrared.

Far-infrared observations are required because galaxies are dusty. Dust is a byproduct of star formation that is essential to astrophysical processes, from planetesimal formation in protoplanetary disks, to radiation-driven galactic outflows. Dust also obscures star formation and SMBH growth, since it efficiently absorbs ultraviolet and visible light, rendering the driving processes of galaxy evolution nearly invisible at these wavelengths. However, the dust re-emits this energy in the far-infrared, making optically dim galaxies brilliant infrared sources. Just as importantly, infrared and molecular emission lines, which directly trace star formation, black hole growth, and metal abundances, can escape dusty galaxies, making the mid- and far-infrared the only bands where a direct, unbiased view of galaxy and metal growth is possible. This lesson was reinforced by Spitzer and Herschel, which provided our first glimpses of dusty galaxies in the infrared during the peak epoch of star formation, when the Universe was only 3 Gyr old. With 1000 times better sensitivity, Origins gives us a clear view of galaxy and metal growth across cosmic time, through a deep-and-wide spectroscopic survey in a wavelength regime inaccessible from the ground and that will remain unexplored by JWST.

How do the stars and supermassive black holes in galaxies evolve with time? Origins uses atomic and molecular emission lines and emission from dust grains to measure the density, temperature, and ionization state of the gas where stars are forming and in galactic nuclei. These observations probe the physics of the interstellar medium, characterize the atomic and molecular gas that drives star-formation, measure the buildup of metals from dying stars, and track the growth of SMBHs and their influence, as they drive energetic outflows into the surrounding interstellar medium (Fig. 3).

How do galaxies make metals, dust, and organic molecules? Galaxies are the metal factories of the Universe, and Origins studies how heavy elements and dust were made and dispersed throughout the cosmic web over the past 12 billion years. Sensitive metallicity indicators in the infrared can be used to track the growth of heavy elements in even the densest optically-obscured regions inside galaxies.

How do the relative energetics from supernovae and quasars influence the interstellar medium of galaxies? Galaxies are made of billions of stars, yet star formation is extremely inefficient on all scales, from single molecular clouds to galaxy clusters. The reason is thought to be 'feedback' from star formation or black hole growth, because supernovae or quasar winds can disrupt star-forming gas. Origins studies the role of feedback processes in galaxies over 
a wide range of environments and redshifts by investigating the processes that drive powerful outflows and surveying the demographics of galactic feedback.

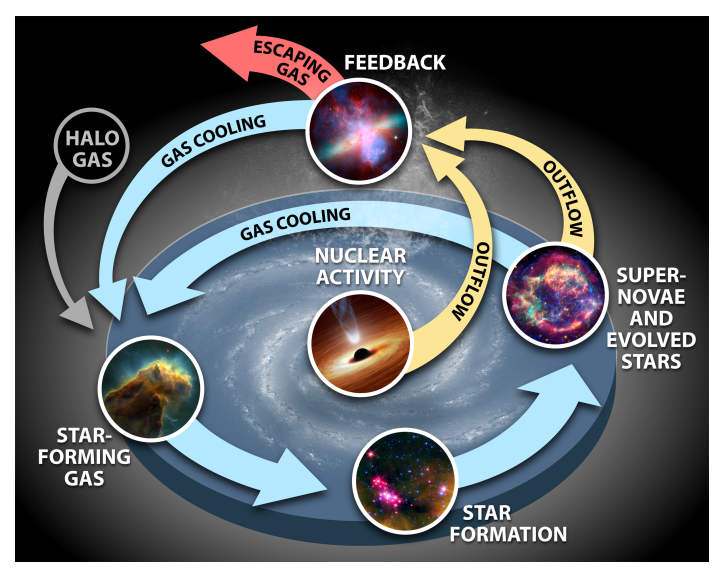

Figure 3. Origins studies the baryon cycle in galactic ecosystems. Energetic processes that shape galaxies and the circumgalactic medium together define this ecosystem. Through its ability to measure the energetics and dynamics of the atomic and molecular gas and dust in and around galaxies, Origins can probe nearly all aspects of the galactic ecosystem: star formation and AGN growth; stellar death; AGN- and starburst-driven outflows; and gas cooling and accretion. These measurements provide a complete picture of the lifecycle of galaxies.

\subsection{How do the conditions for habitability develop during the process of planet formation?}

Water is essential to all life on our planet. Water provides the liquid medium for life's chemistry and plays an essential biochemical role. However, we do not know how terrestrial planets get their water, since rocky planets with liquid water exist in regions where water in icy, protoplanetary dust would have sublimated and photo-dissociated. With its broad wavelength coverage, Origins can detect many water vapor lines that trace the entire range of temperatures found in protoplanetary disks, from the cold snowline to the hot steam line (Fig. 4). Origins can survey all reservoirs of water in more than 1000 planet-forming disks around stars of all masses, including the faint M-dwarfs that likely host most planets in the Galaxy.

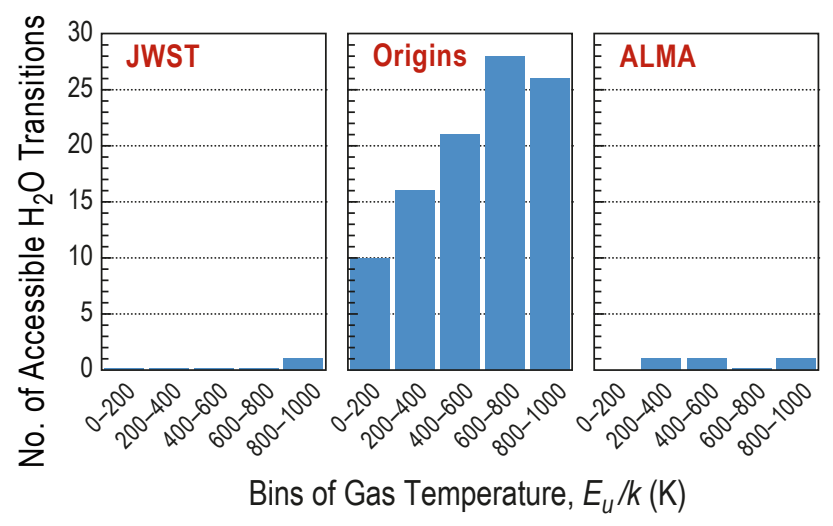

Figure 4. Origins studies more than 100 transitions of water vapor, compared to one and three with JWST and ALMA, respectively. The plot shows the number of $\mathrm{H}_{2}{ }^{16} \mathrm{O}$ transitions observable by JWST, Origins, and ALMA as a function of the gas temperature for energies above the ground state less than $1000 \mathrm{~K}$. ALMA is limited by atmospheric absorption in its ability to observe water.

Another puzzle in planetary formation is the role of hydrogen gas in protoplanetary disks. This gas is the reservoir from which gas giants and planetesimals emerge and the latter are the building blocks of rocky planets. Despite decades of effort, the hydrogen gas mass of protoplanetary disks is essentially unknown because molecular hydrogen, being a symmetric molecule, is largely invisible at the low temperatures of planet-forming gas and the traditional gas mass tracer, CO, is uncertain by factors of 10-100 in disks. Origins can observe the HD $112 \mu \mathrm{m}$ line, which provides a new and robust measure of disk gas mass. 


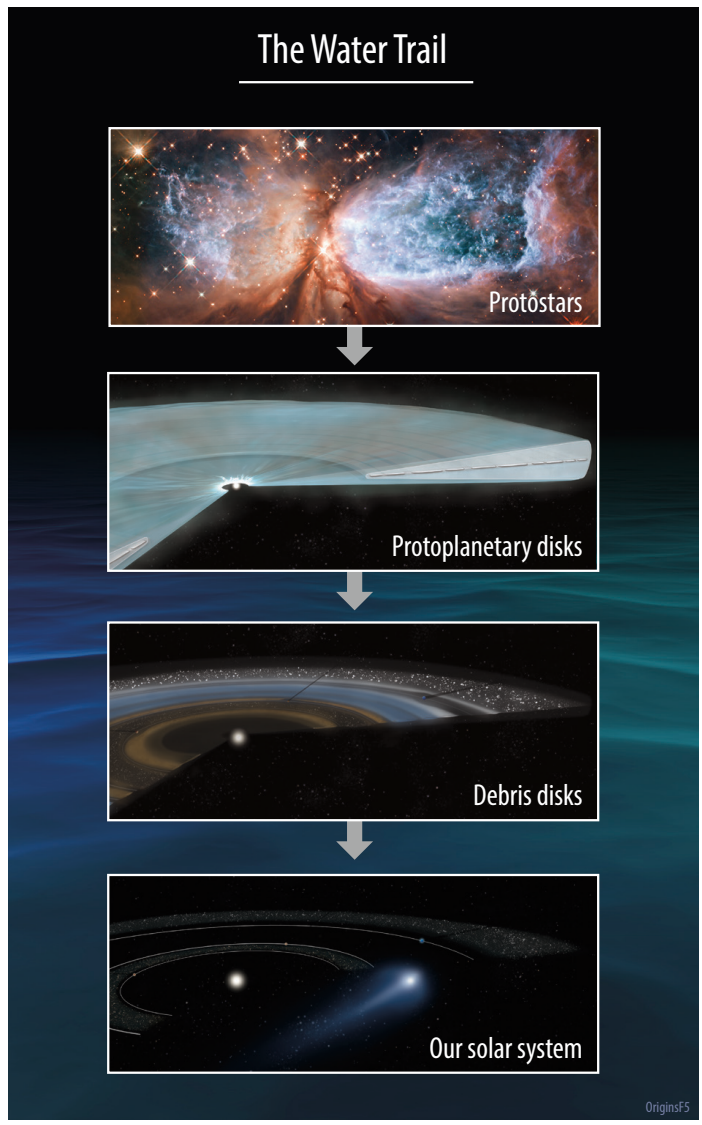

Figure 5. Origins will trace water and gas during all phases of the formation of a planetary system. The trail begins in the "pre-stellar" phase explored by Herschel, where a cloud of gas collapses (top) into a still- forming star surrounded by a disk nearly the size of our Solar System and a collapsing envelope of material $\left(2^{\text {nd }}\right.$ from top). Over time, the envelope dissipates, leaving behind a young star and a disk with nascent planets $\left(3^{\text {rd }}\right.$ from top), eventually leaving behind a new planetary system (bottom). Origins will excel at probing the protoplanetary and later phases.
What role does water play in the formation and evolution of habitable planets? With its unprecedented sensitivity to weak emission from all forms of water (ice as well as gas), Origins deciphers the role of water throughout each phase of planetary system formation (Fig. 5).

How and when do planets form? Origins can uniquely use the HD $112 \mu \mathrm{m}$ emission line, a powerful tool to measure the gas mass of protoplanetary disks to within a factor of 2-3. This precision is one to two orders of magnitude better than alternative tracers and can distinguish between competing models of planet formation and set the timescale for gas giant formation. Origins gas-mass measurements have the potential to provide calibrations for all other observations of protoplanetary disks, including those made with ALMA.

How were water and life's ingredients delivered to Earth and to exoplanets? Earth likely formed within the snowline - the distance from a young star where water transitions from a gas to a solid. Thus, the prevailing theory holds that water was delivered to the early Earth via impacts by bodies that formed beyond the snowline. The evidence for this comes from the high deuterium content of Earth's oceans relative to the protosolar nebula, an excess that is locked when water is formed at a temperature of 1020 K. In our Solar System, comets and asteroids also carry this signature. Only a handful of comets have been measured to date and they show a range of deuterium abundances. With a larger sample of comets, Origins can finally establish if comets were the source of Earth's water.

\subsection{Do planets orbiting M-dwarf stars support life?}

Humankind has long pondered the question, "Are we alone?" Now scientists and engineers are designing instruments dedicated to answering this question. Our quest to search for life on planets around other stars relies on our ability to measure the chemical composition of their atmospheres and understand the data in the context of models for planet formation and evolution. Using the techniques of transmission and emission spectroscopy, Origins will expand upon the legacy of Hubble and Spitzer - and soon JWST - with a mid-infrared instrument specifically designed to characterize temperate, terrestrial exoplanets. In its search for

signs of life, Origins will employ a multi-tiered strategy, beginning with a sample of planets with well-determined masses and radii that are transiting nearby $M$ dwarfs, the most abundant stars in the galaxy. With its broad, simultaneous wavelength coverage and unprecedented stability, Origins will be uniquely capable of detecting life (Figure 6).

What fraction of terrestrial K- and M-dwarf planets has tenuous, clear, or cloudy atmospheres? In the first tier of its exoplanet survey, Origins will obtain transmission spectra over 2.8-20 $\mu \mathrm{m}$ for temperate, terrestrial planets spanning a broad range of planet sizes, equilibrium temperatures, and orbital distances to distinguish between tenuous, clear, and cloudy atmospheres. Because $\mathrm{CO}_{2}$ absorption features are so large, this tier can include terrestrial planets orbiting stars from late-M to late-K, giving Origins a broader perspective in the search for life than JWST.

What fraction of terrestrial M-dwarf planets is temperate? For a subset of planets with the clearest atmospheres, Origins will measure their thermal emission to determine the temperature structure of their atmospheres. This is critical to assessing climate because it yields an understanding of how incoming stellar and outgoing thermal radiation dictate the heating and cooling of the atmosphere. Origins can then determine whether these atmospheric conditions could support liquid water near the surface. 
What types of temperate, terrestrial M-dwarf planets support life? Origins will be the first observatory with the necessary spectroscopic precision to not only measure habitability indicators $\left(\mathrm{H}_{2} \mathrm{O}, \mathrm{CO}_{2}\right)$, but also crucial biosignatures $\left(\mathrm{O}_{3}\right.$ coupled with $\mathrm{N}_{2} \mathrm{O}$ or $\left.\mathrm{CH}_{4}\right)$, which are definitive fingerprints of life on habitable-zone planets. In this observational third tier, Origins will obtain additional transit observations for the highest-ranked targets to search for and detect biosignatures with high confidence. The wavelength range afforded by Origins will provide access to multiple spectral lines for each molecular species. This will increase the detection significances and prevent potential degeneracies due to overlapping features, averting false positives. This framework robustly detects a variety of potentially habitable planet atmospheres, including the life-bearing Archaean Earth. The entire era of exoplanetary science has shown that Nature's imagination trumps our own, and Origins' broad wavelength coverage and precise measurements are guaranteed to give us views into the new and unexpected.

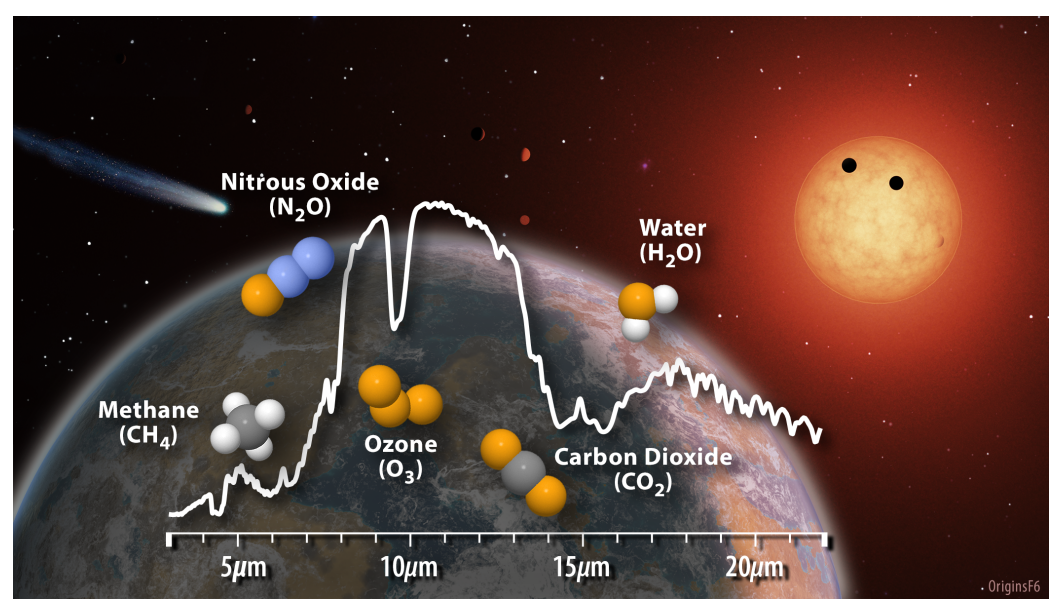

Figure 6. Origins is designed to characterize already-discovered rocky planets that transit $\mathrm{M}$ dwarf stars and place critical constraints on their temperatures. By leveraging the midinfrared wavelengths offered by Origins, these atmospheres can be examined for gases that are the most important signatures of life.

\subsection{Origins: A Mission for the Astronomical Community}

Origins is designed for discovery. While the mission aims to address a specific set of objectives leading to technical requirements, the science program outlined is intended to be illustrative. Origins is a true community observatory, driven by science proposals selected through the usual peer-review process, as used for existing large NASA observatories.

Unanticipated, Yet Transformative, Discovery Space: The Origins-enabled scientific advances described above are extensions of known phenomena. However, history has shown that order-of-magnitude leaps in sensitivity lead to discoveries of unanticipated phenomena. For example, the sensitivity of IRAS over balloon and airborne infrared telescopes led to the discovery of debris disks, protostars embedded within dark globules, Galactic infrared cirrus, and infrared-bright galaxies, none of which were expected at the time of launch. Likewise, no study anticipated that Spitzer would determine the stellar masses of $z>6$ galaxies and characterize the TRAPPIST-1 multi-exoplanet system, the coldest known brown dwarfs, measure winds transporting energy in exoplanet atmospheres, and detect dust around white dwarfs produced by shredded asteroids.

Origins' sensitivity exceeds that of its predecessor missions by a factor of 1000. Jumps of this magnitude are very rare in astronomy, and have always revolutionized our understanding of the Universe in unforeseen ways. Thus, it is essentially guaranteed that the most transformative discoveries of Origins are not even anticipated today.

\section{MISSION DESIGN}

Origins is $>1000$ times more sensitive than prior far-IR missions and the design avoids complicated deployments to reduce mission risk. The scientific objectives summarized in Table 1 are achievable with the low-risk Origins design we propose. Origins has a Spitzer-like architecture (Fig. 7) and requires only a few simple deployments to transform from launch to operational configuration. With the attributes shown in Table 3, the current design carries significant margin between science-driven measurement requirements and estimated performance (Table 2), to ensure success of the science mission. The optical system launches in its operational configuration, requiring no mirror, barrel, or baffle 


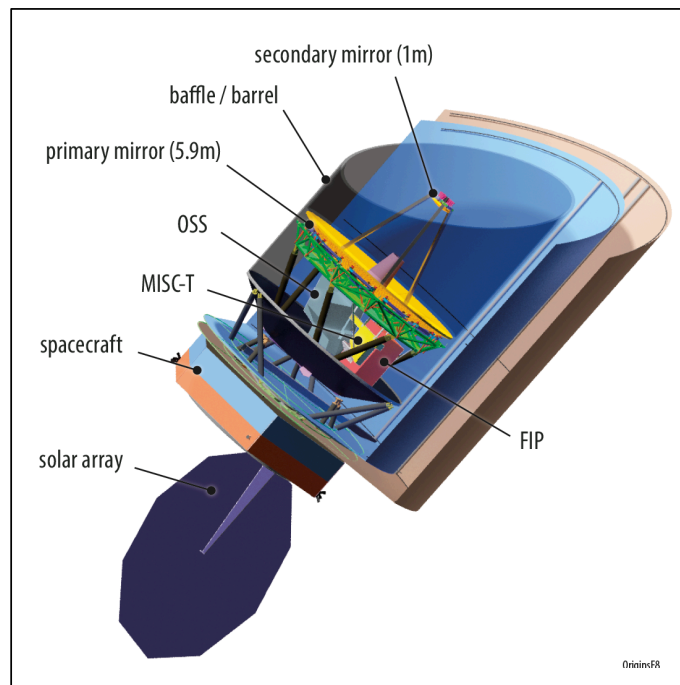

Figure 7. Origins builds on substantial heritage from Spitzer to minimize assembly, integration and testing, and deployment risks. A cutaway view shows the Origins instrument locations and major elements of the flight system.

\begin{tabular}{|l|l|}
\hline \multicolumn{2}{|c|}{ Table 3: Origins Observatory-level Parameters } \\
\hline Mission Parameter & Value \\
\hline Telescope: Aperture Diameter/Area & $5.9 \mathrm{~m} / 25 \mathrm{~m}^{2}$ \\
\hline Telescope Temperature & $4.5 \mathrm{~K}$ \\
\hline Wavelength Coverage & $2.8-588 \mu \mathrm{m}$ \\
\hline Maximum Scanning Speed & $60^{2}$ per second \\
\hline Mass: Dry/Wet (with margin) & $12000 \mathrm{~kg} / 13000 \mathrm{~kg}$ \\
\hline Power (with margin) & $4800 \mathrm{~W}$ \\
\hline Launch Year & 2035 \\
\hline Launch Vehicle (large vehicle) & SLS Block I.B or Space-X BFR \\
\hline Orbit & Sun-Earth L2 \\
\hline Propellant: Lifetime Requirement/Lifetime Goal & 5 years/10 years \\
\hline
\end{tabular}

deployments after launch, but the design allows for mirror segment alignment on orbit to optimize performance. The only deployments are the communication antenna, solar array, telescope cover and sunshields, which are considered low-risk.

This departure from the JWST deployment approach is enabled by the capabilities of new launch vehicles, which are expected to be fully-operational in the mid-2030s. The design is compatible with at least two, and possibly three such launch vehicles. The telescope, is a three-mirror anastigmat with an on-axis secondary. It is diffraction-

limited at $30 \mu \mathrm{m}$ and used as a light bucket at shorter wavelengths, where spatial resolution is not a scientific driver, to perform transit spectroscopy for biosignatures in exoplanets. While Origins has only $2.8 \times$ the collecting area of Herschel, cryo-cooling is the dominant factor affecting its extraordinary sensitivity gain (Fig. 8). To acthieve the same sensitivity gain at optical wavelengths, the light-collecting area would have to increase a thousand-fold. Earth's warm atmosphere limits SOFIA's sensitivity, while a relatively warm telescope $(\sim 80 \mathrm{~K})$ limited Herschel's.

Three science instruments spanning the wavelength range 2.8 to $588 \mu \mathrm{m}$ provide the powerful new spectroscopic and imaging capabilities required to achieve the scientific objectives outlined above (Table 4). The Origins Survey Spectrometer (OSS) uses six gratings to take multi-beam spectra simultaneously across the 25 to $588 \mu \mathrm{m}$ window through long slits enabling deep 3D extra-galactic surveys. When needed, a Fourier transform spectrometer and an etalon provide high and ultra-high spectral resolving power, respectively, especially for studies of water and HD emission lines.

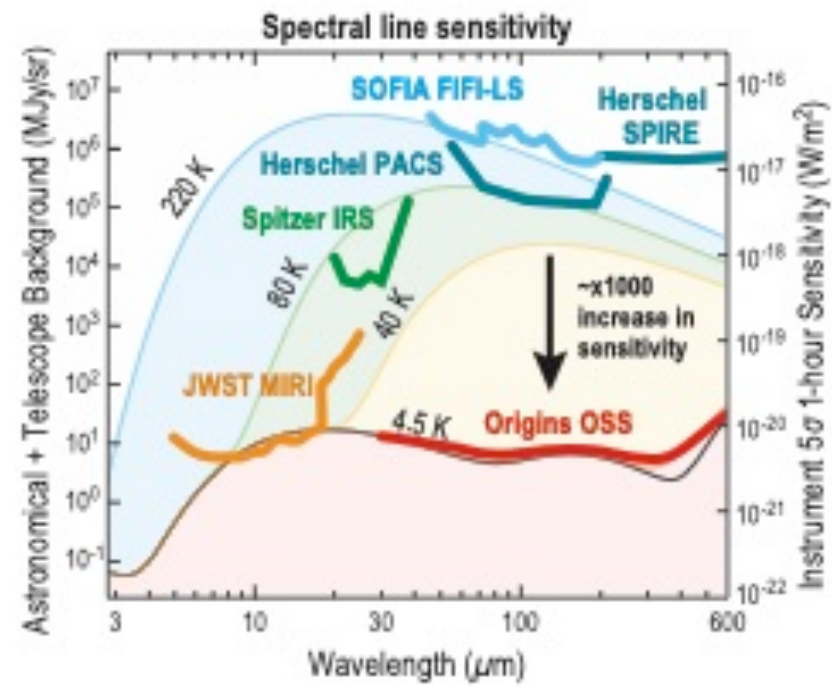

Figure 8. Origins taps into a vast, unexplored scientific discovery space, defined by a three-orders-ofmagnitude improvement in sensitivity relative to all previously-flown far-infrared observatories. With a temperature of $4.5 \mathrm{~K}$, Origins' sensitivity is limited by astronomical background photon noise (lower black curve). SOFIA (220 K), Herschel (80 K), and JWST (40 $\mathrm{K})$ are shown for comparison with Origins (4.5 K). Origins' sensitivity bridges the gap between JWST/MIRI in orbit and ALMA on the ground. 


\begin{tabular}{|c|c|c|c|c|c|}
\hline $\begin{array}{l}\text { Instrument/ } \\
\text { Observing Mode }\end{array}$ & $\begin{array}{l}\text { Wavelength } \\
\text { Coverage }(\mu \mathrm{m})\end{array}$ & Field of View (FOV) & $\begin{array}{l}\text { Spectral Resolving } \\
\text { Power }(R=\lambda / \Delta \lambda)\end{array}$ & $\begin{array}{l}\text { Saturation } \\
\text { Limits }\end{array}$ & $\begin{array}{l}\text { Representative Sensitivity } 50 \text { in } \\
1 \mathrm{hr}\end{array}$ \\
\hline \multicolumn{6}{|c|}{ Origins Survey Spectrometer (OSS) } \\
\hline Grating & $\begin{array}{l}253 / 4588 \mu \mathrm{m} \\
\text { simultaneously }\end{array}$ & $\begin{array}{l}6 \text { slits for } 6 \text { bands: } 2.7^{\prime} \times \\
1.4^{\prime \prime} \text { to } 14^{\prime} \times 20^{\prime \prime}\end{array}$ & 300 & 5 Jy@ $128 \mu \mathrm{m}$ & $3.7 \times 10^{-21}$ W m$m^{-2} @ 200 \mu \mathrm{m}$ \\
\hline High Resolution & $\begin{array}{l}253 / 4588 \mu \mathrm{m} \text { with } \\
\text { FTS }\end{array}$ & Slit: $20^{\prime \prime}$ x [2.7" to 20"] & $43,000{ }^{\prime}[112 \mu \mathrm{m} / \lambda]$ & 5 Jy@ $128 \mu \mathrm{m}$ & $7.4 \times 10^{-21} \mathrm{~W} \mathrm{~m}^{-2} @ 200 \mu \mathrm{m}$ \\
\hline Ultra-High Resolution & $1003 / 4200 \mu \mathrm{m}$ & One beam: $6.7^{\prime \prime}$ & $325,000{ }^{\prime}[112 \mu \mathrm{m} / \mathrm{\lambda}]$ & 100 Jy@ $180 \mu \mathrm{m}$ & $\sim 2.8 \times 10^{-19} \mathrm{~W} \mathrm{~m}^{-2} @ 200 \mu \mathrm{m}$ \\
\hline \multicolumn{6}{|c|}{ Far-IR Imager Polarimeter (FIP) } \\
\hline Pointed & $\begin{array}{l}50 \text { or } 250 \mu \mathrm{m} \\
\text { (selectable) }\end{array}$ & $\begin{array}{l}50 \mu \mathrm{m}: 3.6^{\prime} \times 2.5^{\prime} \\
250 \mu \mathrm{m}: 13.5^{\prime} \times 9^{\prime} \\
(109 \times 73 \text { pixels })\end{array}$ & 3.3 & $\begin{array}{l}50 \mu \mathrm{m}: 1 \mathrm{Jy} \\
250 \mu \mathrm{m}: 5 \mathrm{Jy}\end{array}$ & $\begin{array}{l}\text { 50/250 } \mu \mathrm{m}: 0.9 / 2.5 \mu \mathrm{Jy} \text { Confusion limit } \\
50 / 250 \mu \mathrm{m}: 120 \mathrm{nJy} / 1.1 \mathrm{mJy}\end{array}$ \\
\hline Survey mapping & $\begin{array}{l}50 \text { or } 250 \mu \mathrm{m} \\
\text { (selectable) }\end{array}$ & $\begin{array}{l}60 " \text { per second scan rate, } \\
\text { with above FOVs }\end{array}$ & 3.3 & $\begin{array}{l}50 \mu \mathrm{m}: 1 \mathrm{Jy} \\
250 \mu \mathrm{m}: 5 \mathrm{Jy}\end{array}$ & $\begin{array}{l}\text { Same as above, confusion limit reached } \\
\text { in } 50 / 250 \mu \mathrm{m}: 1.9 \text { hours } / 2 \mathrm{msec}\end{array}$ \\
\hline Polarimetry & $\begin{array}{l}50 \text { or } 250 \mu \mathrm{m} \\
\text { (selectable) }\end{array}$ & $\begin{array}{l}50 \mu \mathrm{m}: 3.6^{\prime} \times 2.5^{\prime} \\
250 \mu \mathrm{m}: 13.5^{\prime} \times 9^{\prime}\end{array}$ & 3.3 & $\begin{array}{l}50 \mu \mathrm{m}: 2 \mathrm{Jy} \\
250 \mu \mathrm{m}: 10 \mathrm{Jy}\end{array}$ & $\begin{array}{l}0.1 \% \text { in linear and circular polarization, } \\
\pm 1^{\circ} \text { in pol. Angle }\end{array}$ \\
\hline \multicolumn{6}{|c|}{ Mid-Infrared Spectrometer Camera Transit Spectrometer (MISC-TRA) } \\
\hline $\begin{array}{l}\text { Ultra-Stable Transit } \\
\text { Spectroscopy }\end{array}$ & $\begin{array}{l}2.83 / 420 \mu \mathrm{m} \text { in } 3 \\
\text { simultaneous bands }\end{array}$ & $\begin{array}{l}2.8-10.5 \mu \mathrm{m}: 2.5^{\prime \prime} \text { radius } \\
10.5-20 \mu \mathrm{m}: 1.7^{\prime \prime} \text { radius }\end{array}$ & $\begin{array}{l}2.8-10.5 \mu \mathrm{m}: 50-100 \\
10.5-20 \mu \mathrm{m}: 165-295\end{array}$ & $\begin{array}{l}\text { k 3.0 mag } \\
30 \text { Jy@3.3pm }\end{array}$ & $\begin{array}{l}\text { Assume K 9.85 mag M-type star, R=50 } \\
\text { SNR/sqrt(hr) }>12,900 @ 3.3 \mu \mathrm{m} \text { in } 60 \\
\text { transits with stability } \sim 5 \mathrm{ppm}<10.5 \\
\mu \mathrm{m}, \sim 20 \mathrm{ppm}>10.5 \mu \mathrm{m}\end{array}$ \\
\hline
\end{tabular}

The Far-IR Imager/Polarimeter (FIP) provides imaging and polarimetric measurement capabilities at 50 and $250 \mu \mathrm{m}$. Its fast mapping enables rapid follow-up of transient or variable sources and efficient monitoring campaigns. FIP surveys take advantage of Origins' agility. Similar to Herschel, Origins can scan-map the sky at 60" per second, which is essential, as the FIP $250 \mu \mathrm{m}$ channel reaches the extragalactic source confusion limit in a few milliseconds. FIP will enable wide area $\left(\geq 1000 \mathrm{deg}^{2}\right)$ photometric surveys, leading to large statistical multiwavelength studies of populations of astronomical objects in wide areas, complementing LSST and WFIRST. Origins will enable the astronomical community to thoroughly explore the currently unknown, faint, far-infrared Universe.

The Mid-Infrared Spectrometer and Camera Transit spectrometer (MISC-T) measures R=50 to 300 spectra in the 2.8 to $20 \mu \mathrm{m}$ range with three bands that operate simultaneously. MISC-T provides exquisite stability and precision for exoplanet transits ( $5 \mathrm{ppm}$ between 2.8 to $10 \mu \mathrm{m}$ ). It employs pupil densification to mitigate observatory jitter and relies on a detector stability improvement relative to current state-of-the-art.

To maximize the sensitivity gain, OSS and FIP incorporate next-generation detectors. As described in section 4, at least two promising detector technologies already exist. Advanced detectors enable Origins to make the first ever fast and wide-area photometric and spatial-spectral surveys in the far-infrared.

In the mid-infrared, from 2.8 to $20 \mu \mathrm{m}$, Origins builds on the amazing discoveries anticipated from JWST. JWST is required to deliver extraordinary sensitivity, but transiting exoplanet spectroscopy was not a major design driver. The Origins team, however, prioritized exoplanet biosignature detection in the important 2.8 to $10 \mu \mathrm{m}$ range, and accordingly established $5 \mathrm{ppm}$ as the required system-level stability for MISC-T. The Origins Technology Development Plan calls for investment in ultra-stable mid-IR detectors and offers multiple parallel development paths to reduce risk.

The Origins design concept minimizes complexity. Origins has a Spitzer-like architecture whose thermal performance is well understood. The solar panel, communication antenna, and telescope cover deployment mechanisms have extensive heritage. The two-layer sunshield deployment relies on stored energy in flexible rods to pull the shield material into its desired shape, and telescoping arms to place each shield at the intended distance from the cold shield (see Fig. 7). This simple deployment sequence can be tested on the ground in existing facilities. The fully-integrated cryogenic payload assembly comprising the telescope, instruments, and cold shield can be tested cryogenically in Chamber A at NASA's Johnson Space Center, following NASA's favored "test-as-you-fly" approach.

The cryo-thermal system design leverages Spitzer experience and technology developed for JWST and Hitomi. Four current-state- of-the-art cryocoolers will cool the telescope to $4.5 \mathrm{~K}$, with $100 \%$ margin in heat lift capacity at each 
temperature stage. The mirrors do not require time-consuming "cryo-null" figuring because the telescope is diffractionlimited at $30 \mu \mathrm{m}$. All of the telescope's mirrors and mirror segments can be diamond-turned and rough-polished to the required precision in existing facilities. The JWST primary mirror segment actuator design is adopted to allow the Origins primary mirror segments to be adjusted in space in three degrees of freedom (tip, tilt, and piston), enabling final alignment during commissioning.

The next generation of launch vehicles, including NASA's SLS, SpaceX's BFR, and Blue Origin's 7m New Glenn, have much larger payload fairings than the 5-m diameter fairings available today, enabling the launch of a largediameter telescope that does not need to be folded and deployed. Origins operates in a quasi-halo orbit around the SunEarth L2 point. The observatory is robotically serviceable, enabling future instrument upgrades and propellant replenishment to extend the mission beyond its 10-year design lifetime.

The Origins mission concept study team worked on a wider set of instrument options than presented in the recommended baseline concept. These options represent possible upscopes which, if adopted, would enhance the mission's scientific capability. These options include the Heterodyne Receiver for Origins (HERO), the MISC Camera, expanded FOVs for OSS and FIP, and additional FIP bands (100 and $500 \mu \mathrm{m})$. The HERO upscope would provide nine-beam spectral measurements of selectable lines in the 110 to $620 \mu \mathrm{m}$ bands up to very high spectral resolving power $\left(\sim 10^{7}\right)$ and could, for example, vastly extend Event Horizon Telescope observations of supermassive black holes. The MISC Camera would enable mid-IR imaging and spectroscopy $(\mathrm{R} \sim 300)$ over the 5 to $28 \mu \mathrm{m}$ wavelength range. The concept study report also discusses potential descopes. In addition to instrument modes, descopes include decreasing the aperture diameter, which impacts the observatory science capabilities as shown in Figure 9.

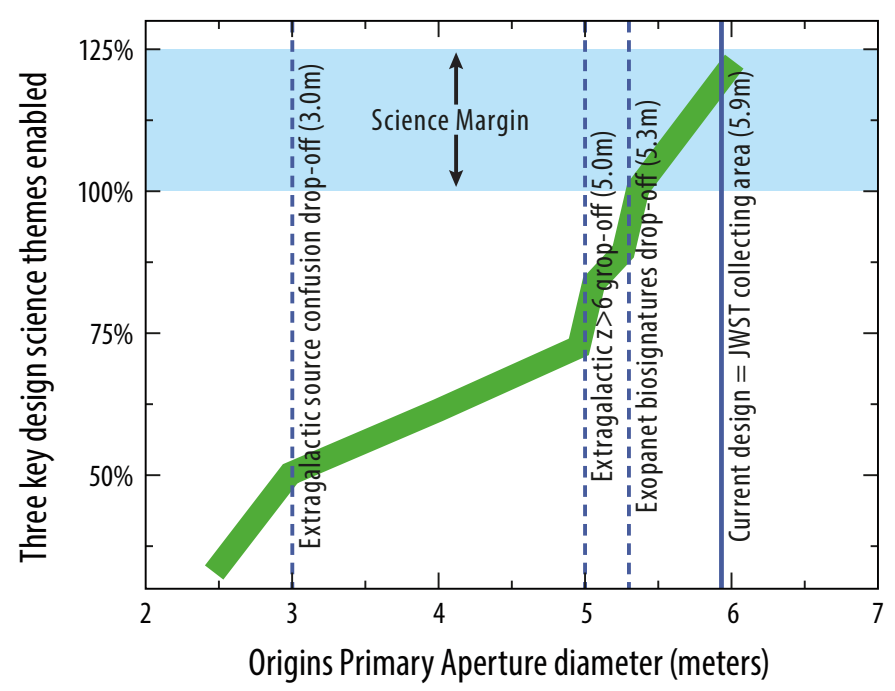

Figure 9. Origins' key science program requires a cold telescope with a primary aperture diameter of $5.3 \mathrm{~m}$. This requirement comes primarily from the exoplanet science case to detect biosignatures in a 5-year mission, given that transit durations are fixed and sensitivity cannot be recovered with a longer single-epoch integration, unlike most other proposed Origins observations. The extragalactic study places an aperture size requirement of $>5 \mathrm{~m}$, based on the need to detect a statistically significant sample of galaxies at $\mathrm{z}>6$, to study the formation mechanisms and physical properties of dust and metals during reionization. The minimum primary aperture diameter is $3 \mathrm{~m}$ to enable an effective extragalactic and Galactic science program, where source confusion does not compromise the telescope's ability to conduct spectroscopic studies of galaxies at $\mathrm{z}=2-3$ and the sensitivity is not too poor to study water and gas in protoplanetary disks at the distances of Orion.

\section{ENABLING TECHNOLOGIES}

Detectors, ancillary detection system components, and cryocoolers are the only Origins enabling technologies currently below Technology Readiness Level (TRL) 5. The Origins Technology Development Plan outlines a path leading to TRL 5 by Phase A start in 2025 and to TRL 6 by mission PDR.

At far-infrared wavelengths, reaching the fundamental sensitivity limits set by the astronomical background requires a cold telescope equipped with sensitive detectors. The noise equivalent power (NEP) required for FIP imaging is $3 \times 10^{-19}$ $\mathrm{W} \mathrm{Hz}^{-1 / 2}$, whereas the NEP needed for OSS for $\mathrm{R}=300$ spectroscopy is $3 \times 10^{-20} \mathrm{~W} \mathrm{~Hz}^{-1 / 2}$. Transition-edge sensor (TES) bolometers and kinetic inductance detectors (KIDs) both show great promise, and we recommend maturation of both technologies to TRL 5 and down-selection to a single technology at the beginning of Phase A (Fig. 10). While the noise requirements for MISC-T's mid- IR detectors are not particularly challenging, 5 ppm stability over several hours must 


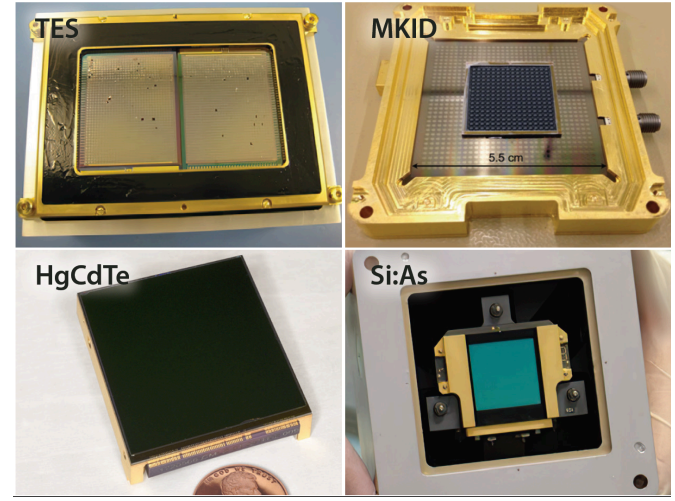

Figure 10. The robust Origins Technology Development Plan recommends parallel detector technologies. TES bolometers (HAWC+ array), KIDS (432-pixel device), HgCdTe arrays (JWST/NIRCam), and Si:As (JWST/MIRI). be demonstrated to meet the Origins requirement. Again, the Origins Technology Development Plan mitigates risk by recommending the parallel maturation of three detector technologies to provide this stability: $\mathrm{HgCdTe}$ and $\mathrm{Si}$ :As arrays and TES bolometers (Fig. 10). An ultra-stable calibration source will be needed for testing.

Mechanical cryocoolers that can reach temperatures of $4.5 \mathrm{~K}$ have already flown on Hitomi (2016). These coolers, developed by Sumitomo Heavy Industries, had a required lifetime of 5 years compared to Origins' 10 years, but meet its performance requirements. Replacing the compressors' suspension system with a flex spring, a relatively straightforward change, is expected to extend the lifetime. Several US companies have also produced TRL 5 cryocoolers or cryocooler components with a projected 10 -year lifetime. The TRL 7 JWST/MIRI cryocooler, for example, has a 6 $\mathrm{K}$ operating temperature. Sub-Kelvin coolers operating at $50 \mathrm{mK}$, as needed for the OSS and FIP detectors, were also flown on Hitomi. A Continuous Adiabatic Demagnetization Refrigerator (CADR) with a much higher cooling power $(6 \mu \mathrm{W}$ vs. $0.4 \mu \mathrm{W}$ for Hitomi $)$ suitable for Origins is currently being developed to TRL 6 under a Strategic Astrophysics Technology (SAT) grant. This new SAT

CADR also demonstrates self-shielding of magnetic fields to $1 \mu \mathrm{T}$, making it compatible with superconducting detectors that demand an ambient field $<30 \mu \mathrm{T}$. A straightforward extension of this ADR technology allows operation at even lower temperatures $(35 \mathrm{mK})$ with similar cooling power. Lowering the operating temperature is a simple way to improve TES detector sensitivity, should that become necessary during mission formulation.

\section{SCHEDULE AND COST}

The Origins team developed a mission design concept, technical approach, technology maturation plan, risk management approach, budget, and a master schedule compatible with NASA guidelines for the Decadal Study and grounded in NASA and industry experience from previous successful large Class A missions.

Origins is a NASA-led mission, managed by a NASA Center, and includes domestic and international partners. The Japanese space agency, JAXA, and a CNES-led European consortium are active participants in the mission concept study, with each contributing an instrument designs. Domestic participants include NASA centers (GSFC, Ames, MSFC), JPL, and industry (Ball Aerospace, Northrop Grumman, Lockheed Martin, Harris Engineering).

Figure 11 shows the Origins Phase A to E schedule from the top level. Scheduled milestones and key decision points are consistent with NASA Procedural Requirements (NPR-7120.5) and formulation and development for Class A missions. The schedule supports an April 2035 launch. The project plan provides 12.7 months of funded schedule reserve along the critical path, exceeding by 1.9 months the required reserve according to Goddard Procedural Requirements 7120.7B "Funded Schedule Margin and Budget Margin for Flight Projects" for Phases C and D (total duration 6.25 years). Much of the design and development work progresses through parallel efforts, and the critical path runs through the most complex instrument, OSS.

Figure 11. Top-level schedule showing key mission milestones culminating in a 2035 launch

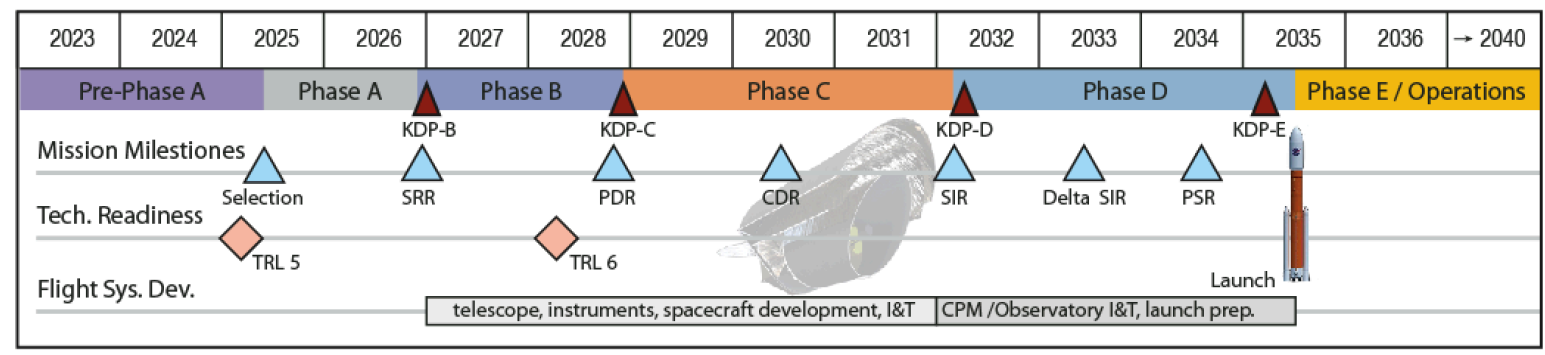


NASA Goddard Space Flight Center's Cost Estimating and Modeling Analysis (CEMA) office developed a cost estimate using the industry standard PRICE-H parametric cost modeling tool. The CEMA cost estimate is based on a detailed master equipment list (MEL) and the Integrated Master Schedule (IMS) for the Origins baseline design. The MEL assigns an appropriate Technology Readiness Level (TRL) to each component. The CEMA cost model assumes that all components have matured to at least TRL 5 by the start of Phase A in 2025, and to at least TRL 6 by mission PDR. A separate Origins Space Telescope Technology Development Plan describes the maturation of all mission-enabling technologies on this timeline and reports the cost of technology maturation. The study team's mission cost estimate includes mission definition and development, the flight segment, the ground segment, and mission and science operations for 5 years. The launch cost $(\$ 500 \mathrm{M}$ for the SLS launch vehicle, as advised by NASA Headquarters) is also included. Working independently, Goddard's Resource Analysis Office (RAO) estimated the mission cost using a topdown parametric model. RAO and CEMA are firewalled from each other, but they both referred to the same MEL and mission schedule. The RAO and CEMA cost estimates agree to within $24 \%$.

Origins is a "large" ( $>\$ 1.5 \mathrm{~B})$ mission using the Decadal Survey's terminology. The NASA Headquarters-appointed Large Mission Concept Independent Assessment Team (LCIT) is tasked with validating the cost estimates supplied by each of the four large missions studied with NASA support. The Origins Final Study Report will provide an LCITvalidated mission cost estimate.

The Origins mission design has not been optimized, and optimization may lead to cost savings. Optimization is planned as a Phase A activity. Japan and several ESA member nations have significant relevant expertise and have demonstrated interest in the Origins mission. Foreign contributions are expected to reduce NASA's share of the mission cost.

\section{SUMMARY}

The Origins Space Telescope was designed to answer three important science questions:

- How do galaxies form stars, make metals, and grow their central supermassive black holes from reionization to today?

- How do the conditions for habitability develop during the process of planet formation?

- Do planets orbiting K and M dwarf stars support life?

The Origins Science and Technology Definition Team (STDT), in consultation with many members of the astronomical community, developed a detailed Science Traceability Matrix (synopsized here) that flows from these questions to three scientific objectives per question, and then to instrument and telescope measurement requirements.

The objectives are achievable with a large (5.9 m diameter) single-aperture telescope cryocooled to $4.5 \mathrm{~K}$, nextgeneration detector arrays, and three science instruments. Taking advantage of new launch vehicle capabilities, the Origins study team developed a low-risk mission concept that borrows its thermal architecture from the highly successful Spitzer Space Telescope, but uses mechanical cryocoolers instead of expendable cryogen. Origins is designed to operate for a minimum of 5 years in a quasi-halo orbit around the Sun-Earth L2 point, with a 10-year mission lifetime goal and an option for robotic servicing to replace instruments and extend the mission beyond 10 years.

To achieve its scientific objectives, Origins will have to be three orders of magnitude more sensitive than any previously flown far-IR telescope, agile enough to enable wide-area imaging and spectroscopic surveys, and its mid-IR instrument will have to be stable enough to measure biosignatures in transiting exoplanets. With access to an observatory having these capabilities, the scientific community will make new discoveries and answer questions that haven't yet been imagined, as well as the profound questions that motivate the mission. In summary, the Origins STDT is prepared to recommend a scientifically compelling, executable mission to the 2020 Decadal Survey. 